\title{
Time-Resolved Photoelectron Spectroscopy Studies of Isoxazole and Oxazole
}

Ting Geng, Johannes Ehrmaier, Oliver Schalk,* Gareth W. Richings, Tony Hansson, Graham Worth, and Richard D. Thomas*

Cite This: J. Phys. Chem. A 2020, 124, 3984-3992

Read Online

ACCESS I

Llll Metrics \& More

Article Recommendations

Supporting Information

ABSTRACT: The excited state relaxation pathways of isoxazole and oxazole upon excitation with UV-light were investigated by nonadiabatic ab initio dynamics simulations and time-resolved photoelectron spectroscopy. Excitation of the bright $\pi \pi^{*}$-state of isoxazole predominantly leads to ring-opening dynamics. Both the initially excited $\pi \pi^{*}$-state and the dissociative $\pi \sigma^{*}$-state offer a combined barrier-free reaction pathway, such that ring-opening, defined as a distance of more than $2 \AA$ A between two neighboring atoms, occurs within 45 fs. For oxazole, in contrast, the excited state dynamics is about twice as slow ( $85 \mathrm{fs}$ ) and the quantum yield for ring-opening is lower. This is caused by a small barrier between the $\pi \pi^{*}$-state and the $\pi \sigma^{*}$-state along the reaction path, which suppresses direct ring-opening. Theoretical findings are consistent with the measured time-

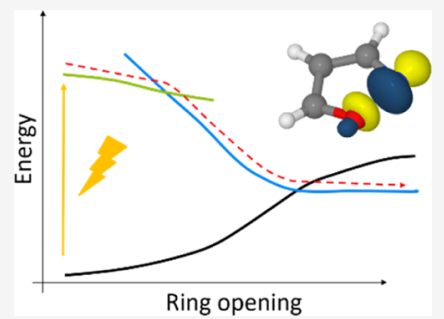
resolved photoelectron spectra, confirming the timescales and the quantum yields for the ring-opening channel. The results indicate that a combination of time-resolved photoelectron spectroscopy and excited state dynamics simulations can explain the dominant reaction pathways for this class of molecules. As a general rule, we suggest that the antibonding $\sigma^{*}$-orbital located between the oxygen atom and a neighboring atom of a five-membered heterocyclic system provides a driving force for ring-opening reactions, which is modified by the presence and position of additional nitrogen atoms.

\section{INTRODUCTION}

The UV-light-induced photodynamics of small heterocyclic aromatic compounds is currently under heavy investigation. This is especially true for the fundamental systems of pyrrole $^{1-6}$ and furan, ${ }^{7-9}$ but to a lesser degree also for their isoelectronic counterparts, which are obtained by replacing a $\mathrm{C}-\mathrm{H}$ unit in the ring by a nitrogen atom. Some common questions that these investigations try to answer are: (i) which are the accessible relaxation channels? (ii) what are the quantum yields of the different channels? and (iii) how fast is the dynamics? All three questions are correlated and cannot be answered independently. In general, the dominant relaxation channels are (a) ring-puckering dynamics, (b) ring-opening dynamics, usually at the heteroatom in the 1-position, and (c) $\mathrm{N}-\mathrm{H}$ bond-breaking in molecules with an $\mathrm{N}-\mathrm{H}$ group. Ringpuckering dynamics can be slow, and the molecule can then get trapped in the excited state long enough to undergo secondary reaction channels, such as intersystem crossing.

Based on these questions, it is possible to dig deeper and pose a more fundamental one: (iv) is it possible to predict the outcome of the photoinduced reaction based on the molecular structure (also known as "Polanyi rules for excited state polyatomic dynamics"10)? In order to make a step toward answering this last question, we performed nonadiabatic excited state $a b$ initio dynamics simulations and time-resolved photoelectron studies on the UV excitation of isoxazole and oxazole (see Figure 1a,c) to fill the gap of missing experiments on these fundamental five-membered heterocyclic systems.
Despite the relatively simple molecular structure, the literature about the photoinduced dynamics of isoxazole is extremely sparse. ${ }^{11-15}$ There is even less literature on the photoinduced dynamics of pure oxazole, although it is more abundant in larger aromatic systems. ${ }^{14,16,17}$ The photochemistry of these compounds in the liquid phase is dominated by the formation of aryl-oxazoles, which are rearrangements of the ring system. ${ }^{18}$ The absorption spectra of isoxazole and oxazole, however, have been studied thoroughly both experimentally and theoretically, and exhibit a broad peak around $6.0 \mathrm{eV}$ indicating a $\pi \pi^{*}$ excitation $(6.0 \mathrm{eV}$ for oxazole and $6.3 \mathrm{eV}$ for isoxazole). ${ }^{19,20}$ Cao et al. studied the photodynamics of isoxazole and oxazole theoretically by using nonadiabatic surface-hopping dynamics simulations based on complete active space self-consistent field (CASSCF) electronic structure calculations. ${ }^{15,17}$ Their calculated vertical excitation energies of the $\pi \pi^{*}$-state are $6.3 \mathrm{eV}$ for isoxazole and $6.7 \mathrm{eV}$ for oxazole, which are in reasonable agreement with experimental observations. ${ }^{15,17}$ Theoretical predictions show that a substantial fraction of the isoxazole molecules undergoes ultrafast $\mathrm{O}-\mathrm{N}$ bond cleavage after photoexcitation, while other

Received: January 2, 2020

Revised: April 3, 2020

Published: April 3, 2020 
(a)

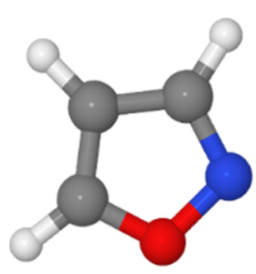

(b)

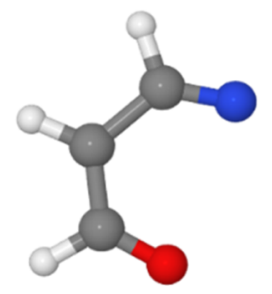

(c)

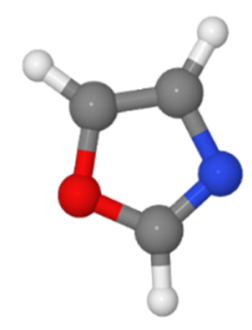

(d)

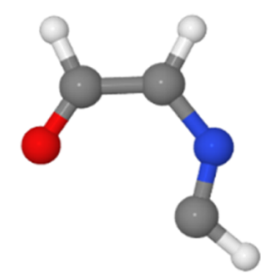

Figure 1. Equilibrium geometries and geometries after ring-opening of isoxazole $(a, b)$ and oxazole $(c, d)$ for the photoinduced ringopening reactions. Ring-opened geometries were obtained by minimizing the energy of the $\pi \sigma^{*}$-state for a bond distance of $3.0 \AA$ using a $\mathrm{C}_{s}$-symmetry constraint. Here, the atoms have the following colors-C (dark grey); O (red); N (blue); and $\mathrm{H}$ (white).

molecules stay intact and return to the ring-closed ground state before possibly undergoing subsequent reactions. ${ }^{\text {i5 }}$ For these latter systems, one such process is the oxazole-isoxazole rearrangement, which has been found in experimental studies for oxazole derivatives. ${ }^{14,21}$ Dias et al. investigated the product yields of isoxazole after photoexcitation at $193 \mathrm{~nm}$ using a chirped-pulse Fourier transform millimeter-wave spectroscopy/pulsed uniform flow technique. ${ }^{13}$ The authors found that the main product channels $\left(\mathrm{HCN}+\mathrm{CH}_{2} \mathrm{CO}\right.$ and $\mathrm{HCO}+$ $\mathrm{CH}_{2} \mathrm{CN}$ with quantum yields of $(53.8 \pm 1.7)$ and $(23.4 \pm$ $6.8) \%$, respectively) were populated because of an ultrafast, nonstatistical, and direct reaction, while minor channels were populated by a statistical distribution from the ground state. The photoinduced dynamics of oxazole has primarily been studied theoretically. ${ }^{16,17} \mathrm{Cao}$ and coworkers found that the main reaction channel is bond cleavage of the $\mathrm{OC}[\mathrm{N}]$-bond after photoexcitation. Molecules are initially excited to the $\pi \pi^{*}$-state, and, within tens of femtoseconds, they are thought to decay to an $n \pi^{*}$-state $\left(S_{1}\right.$ state $)$. Subsequently, most of them undergo bond cleavage, which triggers a hop from the $S_{1}$ to the $\mathrm{S}_{0}$ state.

In the previous descriptions of the photoinduced dynamics of isoxazole and oxazole, the role of the $\pi \sigma^{*}$-state has not been studied in detail. However, it has been shown for the isoelectronic furan ${ }^{9}$ that the $\pi \sigma^{*}$-state, involving the $\sigma^{*}$-orbital at the oxygen atom, plays an important role in the photodynamics, especially with respect to bond cleavage. The antibonding $\sigma^{*}$-orbital of the oxygen atom is also present in both isoxazole and oxazole. Thus, the $\pi \sigma^{*}$-state may play a decisive role in the dynamics in isoxazole and oxazole, as it does in furan. To solve this puzzle, we studied the photodynamics of isoxazole and oxazole molecules through time-resolved photoelectron spectroscopy and excited state nonadiabatic $a b$ initio calculations. The combination of the experimental and theoretical results provides a clear picture of the photoinduced reaction mechanisms for the ring-opening reactions of the two molecules.

\section{METHODS}

2.1. Experimental Methods. Oxazole and isoxazole were purchased from Sigma-Aldrich with purities of 98 and 99\%, respectively, and used without further purification.

The absorption spectra of the molecules taken in a $1 \mathrm{~cm}$ quartz cuvette (Hellma) under saturated vapor pressure were measured with a Cary 5e photospectrometer (Varian). The time-resolved photoelectron spectrometer, a magnetic bottle type apparatus, and other experimental conditions were identical to those described previously. ${ }^{22,23}$ In brief, we used pulses with wavelengths centered at $200 \mathrm{~nm}$ as pump pulses, and pulses centered around $267 \mathrm{~nm}$ as probe pulses. These pulses were generated via frequency quadrupling and tripling of pulses generated by a standard femtosecond laser system (a Coherent Legend USP-HE, producing $800 \mathrm{~nm}$ pulses of $50 \mathrm{fs}$ width). The intensity of the pulses in the interaction region of the magnetic bottle spectrometer was $400 \mathrm{~nJ} /$ pulse for the pump, and $2 \mu \mathrm{J} /$ pulse for the probe. The energy resolution of the experiment was $25 \mathrm{meV}$ and the cross-correlation in the experimental chamber was $140 \pm 10 \mathrm{fs}$, as measured by the nonresonant ionization signal of xenon, which also served as energy calibration for the spectrometer. Sample molecules were injected vertically into the reaction chamber via a gas needle. The temporal delay between the pump and probe pulses was set by a motorized translation stage. At each time delay, the pump-probe signal was corrected by subtracting the background from the pump and probe pulses alone.

2.2. Data Fitting. In order to extract more detailed information, the photoelectron spectra were fitted according to a Levenberg-Marquart 2D global fitting scheme, ${ }^{10}$ which is expressed as

$$
S(E, \Delta t)=\sum_{i} A_{i}(E) P_{i}(\Delta t) \otimes g(\Delta t)
$$

where $A_{i}(E)$ is the decay associated spectrum (DAS) of the channel $\mathrm{i}$, which has a time-dependent population $P_{i}(\Delta t)$, expressed in terms of exponential functions $\exp \left\{-\Delta t / \tau_{i}\right\}$. Here, $\tau_{i}$ is the time constant of the respective process, and $\Delta t=$ $t_{\mathrm{d}}-t_{0}$ is the time delay between the pump and the probe pulses, which overlap at time zero $\left(t_{0}\right)$. Finally, $g(\Delta t)$ is the cross-correlation function obtained from the photoelectron spectrum of Xe (see Experimental Methods section). In order to account for large amplitude motion, that is, when the photoelectron kinetic energies decrease upon pump-probe delay because of a fast decay of the potential energy of the molecule in the excited state and a concomitant rise of the ionization potential, time zero can be artificially used as a fitting parameter. ${ }^{24-26}$ The timescale of the shift can often be used to account for the time a wavepacket needs to reach a conical intersection with a lower-lying potential energy surface. $^{25,26}$ In total, the timescale of the shift and the time constant at the maximum shift provide a measure of the lifetime of the excited state and values that can be directly compared with ab initio trajectory calculations. ${ }^{27}$ In agreement with ref 9 , the uncertainty of the time zero fit is \pm 10 fs in the one-photon pump-one-photon probe region and $\pm 15 \mathrm{fs}$ in the two-photon probe region.

2.3. Computational Methods. Geometries of isoxazole and oxazole were obtained by optimizing the energy of the electronic ground-state using second-order Møller-Plesset 
perturbation theory (MP2). ${ }^{28}$ Vertical excitation energies were subsequently calculated with the second-order algebraicdiagrammatic construction scheme $(\mathrm{ADC}(2)) .{ }^{29}$ To investigate the ring-opening reactions, scans were performed along the $\mathrm{ON}$ - and $\mathrm{OC}[\mathrm{N}]$-distances for isoxazole and oxazole, respectively. The geometries for $\mathrm{ON}$ - and $\mathrm{OC}[\mathrm{N}]$-distances $\geq$ $1.7 \AA$ were obtained by minimizing the energy of the $\pi \sigma^{*}$-state $\left(\mathrm{S}_{1}\right.$-state $)$ at the $\operatorname{ADC}(2)$ level. The OC[N]-bond describes the OC-bond involving the $\mathrm{C}$-atom between the $\mathrm{O}$ - and $\mathrm{N}$ atoms. All internal degrees of freedom except the ON- or OCdistance were relaxed to minimize the energy. In order to be able to select the $\pi \sigma^{*}$-state, the $C_{s}$ symmetry constraint had to be applied in the static calculations. Despite the fact that it limits the configurational space, it is assumed that the calculations provide a clear qualitative picture of the systems. Ground-state energies were calculated at the MP2 level at the optimized geometries. The geometries for the scans from the Franck-Condon (FC) geometry to the geometry optimized for the energy of the $\pi \sigma^{*}$-state at an $\mathrm{ON}$ - or OC $[\mathrm{N}]$-distance of $1.7 \AA$ were constructed by linear interpolation of Cartesian coordinates. Ground-state and excited state energies were calculated as single-point calculations at the MP2 and ADC(2) levels at the given geometries without further optimization.

The photoinduced dynamics of isoxazole and oxazole was studied using on-the-fly nonadiabatic surface-hopping simulations. In these direct-dynamics simulations, Newton's equations of motion for the nuclei are solved using electronic energies and gradients calculated at the $\operatorname{ADC}(2)$ level for each step without any constraints. A Landau-Zener-based surfacehopping algorithm was used to account for nonadiabatic transitions of the trajectories between different electronic states: a computationally efficient approach has been recently implemented in the direct-dynamics surface-hopping code of the group of Došlic ${ }^{30}$ and has been benchmarked carefully. ${ }^{31,32}$ The lowest three excited states and the ground-state were included in the calculation. Starting geometries for the trajectories were obtained by Wigner sampling, and molecules were initially placed on the $S_{1}$-state. In total, 100 trajectories were propagated for $100 \mathrm{fs}$ with a time step of $0.5 \mathrm{fs}$ for each system. All electronic structure calculations were performed with the Turbomole program package ${ }^{33}$ applying the resolution of identity approximation and Dunning's augmented correlation consistent double zeta (aug-cc-pVDZ) basis set. ${ }^{34}$

\section{RESULTS}

3.1. Computational Results. We performed static and dynamical simulations on the photoinduced reaction pathways of isoxazole and oxazole, thereby focusing on the ring-opening reactions of the two molecules. Figure 1a,c show their equilibrium geometries optimized at the MP2 level. The ON-distance of the optimized geometry of isoxazole is $1.380 \AA$, which is slightly shorter than the experimentally observed equilibrium bond distance of Stiefvater, which is $1.399 \AA^{35}$ The OC $[\mathrm{N}]$-distance in oxazole, that is the OC-bond including the $\mathrm{C}$-atom between the oxygen and nitrogen atoms, is a little bit shorter and measures $1.367 \AA$, which is in between Cao's calculated result of $1.371 \AA^{17}$ and the experimentally measured distance of $1.357 \AA^{36}$ The OC $[\mathrm{N}]$ bond is prone to ring-opening after photoexcitation, as shown in Figure 1d.

Vertical excitation energies at the ground-state equilibrium geometry with the corresponding oscillator strengths are summarized in Table 1 . For both molecules, the $S_{1}$-state is
Table 1. Vertical Excited State Energies in $\mathrm{eV}$ and Oscillator Strengths in Parentheses for Isoxazole and Oxazole Calculated at the $\operatorname{ADC}(2) /$ aug-cc-pVDZ Level of Theory

\begin{tabular}{ccc} 
state & isoxazole & oxazole \\
$\mathrm{S} 1\left(2 \mathrm{~A}^{\prime}, \pi \pi^{*}\right)$ & $6.53(0.0926)$ & $6.41(0.1584)$ \\
$\mathrm{S} 2\left(1 \mathrm{~A}^{\prime \prime}, n \pi^{*}\right)$ & $6.62(0.0057)$ & $6.48(0.0031)$ \\
$\mathrm{S} 3\left(2 \mathrm{~A}^{\prime \prime}, \pi \sigma^{*}\right)$ & $6.88(0.0226)$ & $6.51(0.0151)$ \\
\hline
\end{tabular}

of $\pi \pi^{*}$-character and carries significant oscillator strength. The $\mathrm{S}_{1}$-state of isoxazole is at $6.53 \mathrm{eV}$, which is in good agreement with Cao's CASPT2/cc-pVTZ result of $6.33 \mathrm{eV}^{15}$ and Walker's computational result of $6.30 \mathrm{eV}$. Moreover, it coincides reasonably well, considering the accuracy of the method and the zero point energy, with the experimental value of around $6.0 \mathrm{eV}{ }^{19}$ The experimental value is estimated from the maximum of a broad peak, which is assigned as a $\pi \pi^{*}$ excitation because of its bathochromic shift from the gas phase to aqueous solution. The ordering of the three lowest states of isoxazole coincides with the ordering in ref $19 . S_{4}$ is predicted to be a bright valence state at $6.9 \mathrm{eV},{ }^{19}$ which is, however, not included in our study. For oxazole, the lowest $\pi \pi^{*}$-excitation is calculated to be at $6.41 \mathrm{eV}$, and experimentally, the peak of the first broad maximum, which corresponds to the $\pi \pi^{*}$-state, is at $6.2 \mathrm{eV}^{20}$ The second state, $S_{2}$, is of $\mathrm{n} \pi^{*}$-character for both molecules, whereas $\mathrm{S}_{3}$ is a $\pi \sigma^{*}$-state and is called a Rydberg state because it involves a diffuse virtual $\sigma^{*}$-orbital. The oscillator strengths of the $S_{2^{-}}$and $S_{3}$-states are significantly lower than that of $S_{1}$. As a consequence, excitation to the $S_{1}$ state is supposed to be dominant upon irradiation with photons in the $6.2 \mathrm{eV}$ region. In the case of oxazole, our state ordering is consistent with Palmer's computational results (at the multireference multiroot configuration interaction (MRDCI) level with triple-zeta basis sets) that predict excited state energies of $6.00 \mathrm{eV}\left(\pi \pi^{*}\right), 6.34 \mathrm{eV}\left(\mathrm{n} \pi^{*}\right)$, and $6.39 \mathrm{eV}\left(\pi \sigma^{*}\right)$ for $S_{1}, S_{2}$, and $S_{3}$, respectively. ${ }^{20}$ Cao and coworkers, however, found a different ordering at the multistate CASSCF/CASPT2 level, where the lowest lying excited state $\left(S_{1}\right)$ is of $n \pi^{*}$ character $(6.21 \mathrm{eV})$ and the $\mathrm{S}_{2}$-state is the bright $\pi \pi^{*}$-state $(6.72 \mathrm{eV})$ with a conical intersection between the two states. ${ }^{17}$ At the $\mathrm{ADC}(2)$ level with the nonaugmented cc-pVDZ basis set, we obtain the same inverted ordering with the $\mathrm{n} \pi^{*}$ state at $6.77 \mathrm{eV}$ and the $\pi \pi^{*}$ state at $6.92 \mathrm{eV}$. The lower energy of the $\mathrm{n} \pi^{*}$-state can be explained by an overestimation of the energy of the n-orbital, which is caused by the nonaugmented basis set. Therefore, all further calculations have been performed with the aug-cc-pVDZ basis set. Finally, it is worth mentioning that the reactive $\pi \sigma^{*}$ state, which is responsible for the ringopening reactions because of the antibonding orbital of the oxygen atom, is not shown in Table 1. At the FC geometry, it is hidden in a dense set of highly energetic Rydberg states and hard to identify.

In the case of isoxazole, the ON-bond is broken, whereas in the case of oxazole, the dominant reaction is a ring-opening at the $\mathrm{OC}[\mathrm{N}]$-bond. The product geometries of isoxazole and oxazole, where the energies of the reactive $\pi \sigma^{*}$-states were optimized for a bond distance of $3.0 \AA$ applying the $C_{s}$ symmetry constraint, are shown in Figure $1 \mathrm{~b}$ and $d$, respectively. Figure 2 shows scans along the ring-opening reaction coordinate, which is the $\mathrm{ON}$-distance in the case of isoxazole and the $\mathrm{OC}[\mathrm{N}]$-distance for oxazole. The scans consist of two parts. For large distances $(\geq 1.7 \AA)$, the geometries were obtained by minimizing the energy of the 
(a)

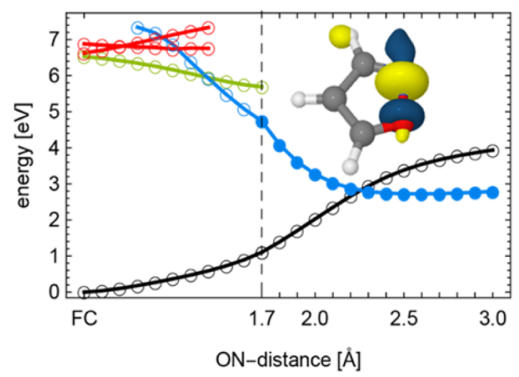

(b)

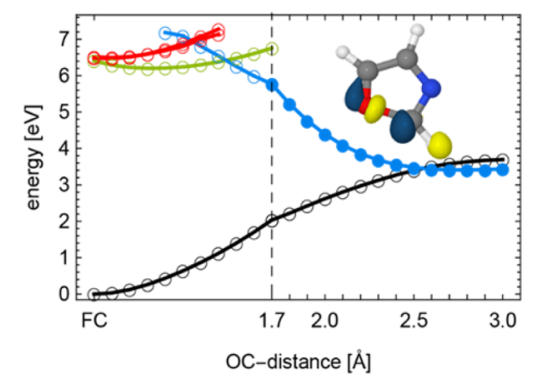

Figure 2. Scans along the ring-opening reaction coordinate. The geometries were obtained by optimizing the energy of the reactive $\pi \sigma^{*}$-state for ON-distances $\geq 1.7 \AA$. From the FC geometry to the optimized geometry at $1.7 \AA$, the geometries were constructed by linear interpolation. For isoxazole, (a) a complete barrier-free path is obtained starting from the $\pi \pi^{*}$-state at the FC point, whereas for oxazole (b) a small barrier has to be overcome. Black: $\mathrm{S}_{0}$-state; red: $\mathrm{A}^{\prime \prime}$-states $\left(n \pi^{*}\right.$ - and $\pi \sigma^{*}$-states); green: $\pi \pi^{*}$-state, blue: reactive $\pi \sigma^{*}$-state. In the inset, antibonding $\sigma^{*}$-orbitals of (a) isoxazole and (b) oxazole at ON- and OC-bond distances of $1.7 \AA$ are shown.

(a)

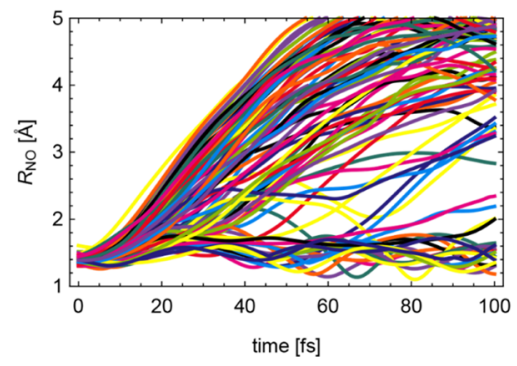

(b)

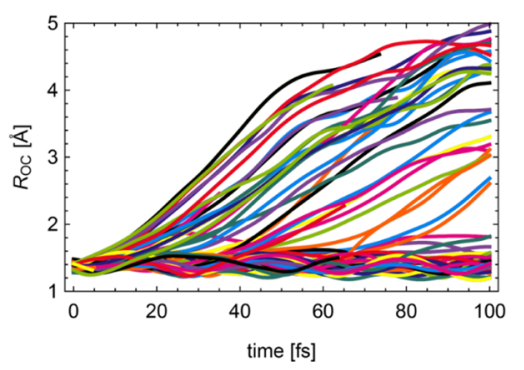

Figure 3. (a) NO-distances of isoxazole and (b) and OC $[\mathrm{N}]$-distances of oxazole for all trajectories upon photoexcitation to the $\mathrm{S}_{1}\left(\pi \pi^{*}\right)$-state during the dynamics.

lowest $\pi \sigma^{*}$-state (blue full dots). The energy of the electronic ground-state configuration (black open circles) was calculated at the obtained geometry. In this region (bond lengths of 1.7$3.0 \AA$ ), the gradient of the $\pi \sigma^{*}$-state points toward larger bond distances, thus providing a driving force for the reaction. Along the reaction coordinate, an intersection between the energy curves of the ground state and the $\pi \sigma^{*}$-state occurs at $2.25 \AA$ $(2.75 \mathrm{eV})$ and $2.55 \AA(3.43 \mathrm{eV})$ for isoxazole and oxazole, respectively. The position of the intersection, the slope of the energy curves at the intersecting point, and the product energies all support a more efficient ring-opening reaction for isoxazole than for oxazole. The virtual $\sigma^{*}$-orbitals at a bond distance of $1.7 \AA$, which get occupied in the $\pi \sigma^{*}\left(\mathrm{~S}_{1}\right)$ electronic configuration, are shown in the inset of Figure 2. Occupation of the orbitals leads to a destabilization of the ONor OC $[\mathrm{N}]$-bond, but the antibonding nature is more strongly developed in the case of isoxazole (Figure $2 \mathrm{a}$ ) compared to the virtual $\sigma^{*}$-orbital of oxazole (Figure $2 \mathrm{~b}$ ). The antibonding character of the $\sigma^{*}$-orbitals explains the large driving force in the $\pi \sigma^{*}$-state toward large distances, and the more favorable potential energy surfaces for isoxazole, as shown in Figure 2.

The relaxed scans for large distances $(\geq 1.7 \AA)$ are minimum energy paths for the ring-opening reaction driven by the reactive $\pi \sigma^{*}$-state. However, after photoexcitation of isoxazole or oxazole, the systems are most likely in the $\pi \pi^{*}$-state in the FC region because of its large oscillator strength. To connect the FC region with the minimum energy path for large bonddistances, we constructed a scan from the ground-state equilibrium geometry to the geometry optimized for the energy of the $\pi \sigma^{*}$-state at a bond distance of $1.7 \AA$ (left side of the dashed line in Figure 2). It is worthwhile noting that these scans only describe a bond elongation of about $0.3 \AA$ (though 9 intermediate points were calculated) from the FC geometries. These scans provide details of the potential energy surfaces in the FC region and show how to reach the reactive $\pi \sigma^{*}$-state after photoexcitation. For isoxazole, the gradient of the $\pi \pi^{*}$-state, depicted in green in Figure $2 \mathrm{a}$, points monotonously toward large ON distances, and generates a net driving force for the ring-opening reaction. The potential energy surface intersects with the energy curve of the $\pi \sigma^{*}$-state (blue curve) at $5.9 \mathrm{eV}$, which is $0.6 \mathrm{eV}$ below the vertical excitation energy. From this scan (Figure 2a), we expect an easily accessible conical intersection between the $\pi \pi^{*}$ - and $\pi \sigma^{*}$-state, which allows efficient population transfer between the states. In the case of oxazole, shown in Figure $2 b$, the $\pi \pi^{*}$ state exhibits a shallow minimum along the chosen reaction path from the FC point to the geometry optimized at an OCdistance of $1.7 \AA$. The intersection between the energy curve of the $\pi \pi^{*}$-state and the $\pi \sigma^{*}$-state is at $6.42 \mathrm{eV}$, which is as high as the vertical excitation energy. Similar to isoxazole, the reactive $\pi \sigma^{*}$-state is accessible in oxazole after photoexcitation but, because of the minimum of the $\pi \pi^{*}$-state close to the FC region and the barrier before the intersecting point, a lessefficient transition to the reactive $\pi \sigma^{*}$-state is expected.

100 trajectories were propagated for 100 fs starting from the $\mathrm{S}_{1}$-state in order to simulate the dynamics of the two molecules after photoabsorption. To monitor the ring-opening reaction, the $\mathrm{ON}$ - and $\mathrm{OC}[\mathrm{N}]$-distances in isoxazole and oxazole were analyzed as a function of the time after excitation. The results are plotted in Figure $3 a$ for isoxazole and in Figure $3 b$ for oxazole. If the bond distance increases to more than $2.0 \AA$, the trajectory is recorded as a "ring-opening trajectory". In the case 
(a)

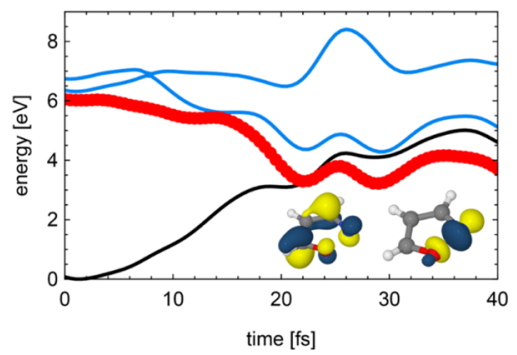

(b)

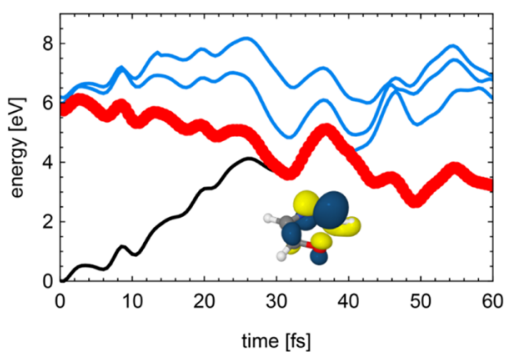

Figure 4. Energy profiles for two representative trajectories of (a) isoxazole and (b) oxazole undergoing ring-opening. Black: $\mathrm{S}_{0}$; blue: excited states; red dots: the electronic state of the trajectory. Insets show the virtual $\pi^{*}$-orbital of the bright $\pi \pi^{*}$-state and virtual $\sigma^{*}$-orbital of the reactive $\pi \sigma^{*}$-state of the representative trajectory of isoxazole at $t=14 \mathrm{fs}(\mathrm{a})$, and the virtual orbital of the $S_{1}$-state of the representative trajectory of oxazole at $t=15$ fs (b).

of isoxazole, more than $80 \%$ of the trajectories show ringopening within the first 100 fs. Only for very few cases, a ringopening and subsequent ring-closing reaction is observed, suggesting that ring-opening is a rarely reversible relaxation channel. For oxazole, both the quantum yield for ring-opening is lower and the process is slower. Here, only $40 \%$ of all the trajectories show a ring-opening reaction. We also refer to Figure S2 in the Supporting Information for a summary of the opening probability for all trajectories over time. Already during the first $100 \mathrm{fs}$, a substantial difference in the photochemical reactions between the two molecules becomes apparent, which is consistent with the findings of our scans that the potential energy surfaces of the $\pi \pi^{*}$-states differ qualitatively in the FC region. The topology of the potential energy surfaces of the $\pi \pi^{*}$ and $\pi \sigma^{*}$-states is responsible for the differences in the dynamics of the molecules, which will become clear by analyzing the representative trajectories.

Figure $4 a, b$ show the energy profiles for representative ringopening trajectories of isoxazole and oxazole, respectively. The ground-state energy curve is shown in black, excited state energy curves are plotted in blue, and red dots indicate the state of the trajectory at a given time. For isoxazole, the trajectory starts in the $S_{1}\left(\pi \pi^{*}\right)$-state. After $t=14 \mathrm{fs}$, the lowest two excited electronic states are almost degenerate, which correspond to the $\pi \pi^{*}$ - and the reactive $\pi \sigma^{*}$-state. In the inset of Figure 4, the dominant virtual orbitals of the $S_{1}$ - and $S_{2}$ states at $t=14 \mathrm{fs}$ are depicted. They are similar to the $\pi^{*}$ and the antibonding $\sigma^{*}$-orbital of the bright $\pi \pi^{*}$-state and the reactive $\pi \sigma^{*}$-state (cf. Figure $2 \mathrm{a}$ ). The trajectory continues on the surface of the $S_{1}$-state, whose character changes at this point to the reactive $\pi \sigma^{*}$-state. Because the $S_{1^{-}}$and $S_{2}$-states are almost degenerate, and because of the change of the electronic character of the states, we expect a close lying conical intersection. At about $22 \mathrm{fs}$, a curve crossing of the $S_{1}$ state and the ground state occurs at $3.4 \mathrm{eV}$. The trajectory continues on the surface of the $\pi \sigma^{*}$-state, which becomes the most stable configuration. Thus, the dynamics confirms that, after photoexcitation to the $\pi \pi^{*}$-state, the system can effectively be transferred to the reactive $\pi \sigma^{*}$-state by a conical intersection, which then becomes the most stable configuration for large bond distances.

Figure $4 b$ shows the potential energy curves for a representative trajectory of oxazole undergoing ring-opening. For this particular trajectory, the OC-bond starts to stretch immediately and the ring opens after around $20 \mathrm{fs}$. Along the bond cleavage coordinate, a large increase of the ground-state energy occurs, whereas the energy of the $S_{1}$-state drops. There is, however, no point of degeneracy between the $S_{1}$ - and the $S_{2}$ state along the opening process, which is qualitatively different compared to isoxazole. When considering the occupied virtual orbital of the $S_{1}$-state at $t=15 \mathrm{fs}$, shown in Figure $4 \mathrm{~b}$, we observe that part of the orbital resembles the $\pi^{*}$-orbital of the bright $\pi \pi^{*}$-state, but electronic density is also located between the $\mathrm{O}$ - and $\mathrm{C}$-atom close to the $\mathrm{C}$-atom. This part of the orbital is similar to the corresponding density of the antibonding $\sigma^{*}$-orbital of the reactive $\pi \sigma^{*}$-state shown in Figure $2 \mathrm{~b}$. Together with the fact that there is no point of degeneracy, we conclude that the trajectory does not come very close to a conical intersection, but that an adiabatic transition takes place. The character of the $S_{1}$-state transforms from the bright $\pi \pi^{*}$-state to the reactive $\pi \sigma^{*}$-state along the trajectory. We suggest that the reduced efficiency of the ringopening reaction in oxazole is caused by the reduced accessibility of the $S_{2} / S_{1}$ conical intersection, corresponding to the intersection between the blue and the green curve in Figure 2.

3.2. Photoelectron Spectrum of Oxazole. The timeresolved photoelectron spectrum of oxazole and isoxazole, pumped with $200 \mathrm{~nm}(6.23 \mathrm{eV})$ and probed with $267 \mathrm{~nm}$ $(4.66 \mathrm{eV})$, is plotted in Figures 5 and 6, respectively. According to ref 20, the adiabatic ionization potential of oxazole is 9.66 $\mathrm{eV}$ while the vertical ionization potential is $\sim 9.82 \mathrm{eV}$. The expected energy cut-off for a one-photon pump, one-photon probe experiment (further denoted as $\left[1,1^{\prime}\right]$ ) is thus around
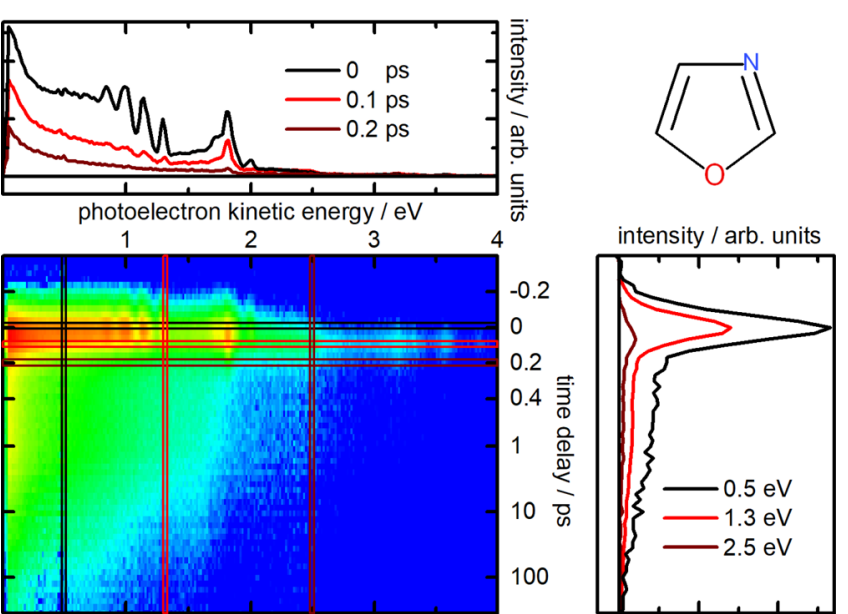

Figure 5. Time-resolved photoelectron spectrum of oxazole upon excitation at $200 \mathrm{~nm}$ and probed at $267 \mathrm{~nm}$. 


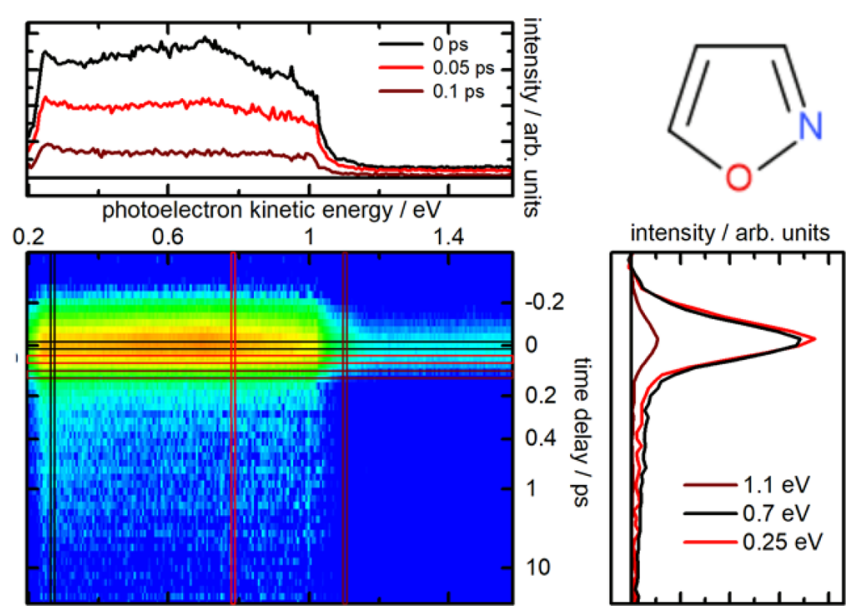

Figure 6. Time-resolved photoelectron spectrum of isoxazole upon excitation at $200 \mathrm{~nm}$ and probed at $267 \mathrm{~nm}$.

$1.23 \mathrm{eV}(6.23+4.66 \mathrm{eV}-9.66 \mathrm{eV}=1.23 \mathrm{eV})$. The photoelectron spectrum consists of four distinct, close lying peaks around the $\left[1,1^{\prime}\right]$ cut-off, which are caused by a vibronic progression in the ionic state. The ionization potential refers to the peak with the second lowest energy of the photoelectron spectrum, which agrees with the observed energy cut-off of about $1.2 \mathrm{eV}$ (see Figure 7a). In addition, we note a prominent

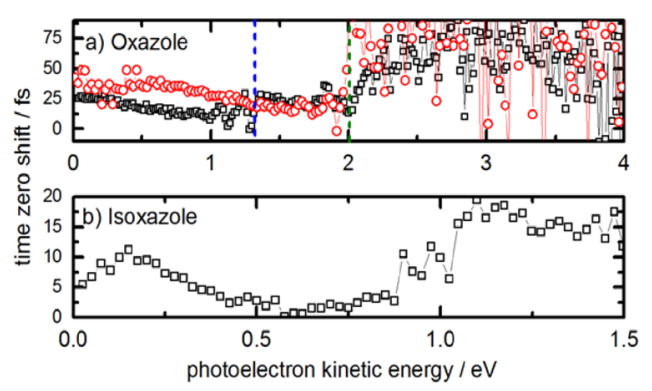

Figure 7. Time zero shifts for (a) oxazole and (b) isoxazole for excitation at $200 \mathrm{~nm}$ and probed at $267 \mathrm{~nm}$. In (a) the time zero shift of furan (200 nm pump and $267 \mathrm{~nm}$ probe) is also shown with red open circles, and the blue dashed line in (a) indicates the $\left[1,1^{\prime}\right]$ cutoff for oxazole, while the green-dashed line indicates the $\left[1,1^{\prime}\right]$ cut-off for furan.

peak at around $1.8 \mathrm{eV}$ in the $\left[1,2^{\prime}\right]$ region, which likely originates from ionization through the 3s-Rydberg state, that is accessing the 3s Rydberg state by one $267 \mathrm{~nm}$ photon and probed by the second $267 \mathrm{~nm}$ photon. The quantum defect in this case would be 0.83 . More peaks originating from ionization through Rydberg states can be observed in the two-photon probe region. These states are not of major interest for this publication, and we refer to ref 27 for more details on the calculations and the discussion on these states.

The best fitting of the data was achieved using a triexponential decay function plus a shifting time zero (see Section 2.2). The time constants were $(25 \pm 10) \mathrm{fs},(2 \pm 0.4)$ ps, and $(65 \pm 10)$ ps. An additional infinite time constant was essentially zero. The time zero shift progresses to $25 \mathrm{fs}$ in the $\left[1,1^{\prime}\right]$ region, but stretches further to $(60 \pm 10)$ fs in the $\left[1,2^{\prime}\right]$ region (see Figure 7a). This additional stretch can be considered as a continuation of the time zero shift observed in the $\left[1,1^{\prime}\right]$ region signal because two probe photons allow us to access states with lower potential energy because of the extra energy provided by the second photon. The stretch and the first time constant are similar to the isoelectronic furan (the time zero shift is added to Figure $7 \mathrm{a}$ ), where the signal was assigned to ring-opening dynamics. ${ }^{8,9}$ The timescale of the dynamics also roughly matches the computational results. We note that the $25 \mathrm{fs}$ time constant has no relevant meaning because it is only a measure for a rapidly moving wavepacket (see Section 2.2), and the relevant time constant is the shift plus the time zero shift, which totals $85 \mathrm{fs}$.

In addition to the short time scale, there is a significant part of the signal that can be observed during an extended time period of several tens of picoseconds. This signal is unstructured that is a sign of a dephased wavepacket (see the DAS in Figure 8a). Because ground state molecules

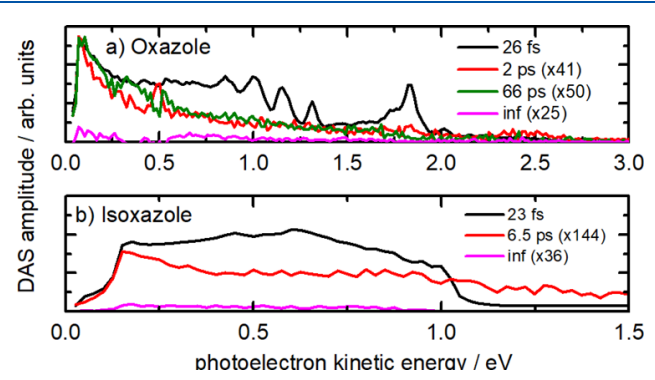

Figure 8. Decay associated spectra of (a) oxazole and (b) isoxazole. For clarity, the amplitude of a longer time constant was given by different factors.

typically do not exhibit any significant signal in the timeresolved photoelectron spectra when using wavelengths in the UV region, ${ }^{38}$ the signal must originate from the excited state molecules. This is a clear sign that a major fraction of the molecules do not undergo prompt excited state ring-opening, which is in agreement with our theoretical results. The origin of the signal is discussed in Section 4.

3.3. Photoelectron Spectrum of Isoxazole. The vertical ionization potential of isoxazole is $10.12 \mathrm{eV}$, with an adiabatic ionization potential of $\sim 9.94 \mathrm{eV},{ }^{19}$ and the progression is spaced more narrowly than in oxazole (the two progressions seen in isoxazole are 550 and $1480 \mathrm{~cm}^{-1}$, as compared to 1160 and $1360 \mathrm{~cm}^{-1}$ in oxazole ${ }^{19,37}$ ) such that no structure is expected in the photoelectron spectrum. The expected $\left[1,1^{\prime}\right]$ cut-off is thus at $10.89-9.94 \mathrm{eV}=0.95 \mathrm{eV}$. The photoelectron spectrum shows a relatively broad band spectrum reaching over the energy cut-off. However, a significant rise of the spectrum is observed around the energy cut-off, and a change of the time zero fit at the same energetic position confirms the cut-off energy. The time zero shift is $10 \mathrm{fs}$ in the $\left[1,1^{\prime}\right]$ region, and $20 \mathrm{fs}$ when the $\left[1,2^{\prime}\right]$ region is included (see Figure $7 \mathrm{~b}$ ), and the fitted time constants of isoxazole are $25 \mathrm{fs}$ and $6.5 \mathrm{ps}$ (see Figure $8 \mathrm{~b}$ ). The fast time component has a dominant amplitude, which is $>100: 1$ greater than the slower time constant throughout the whole spectrum. This suggests that the process associated with this time constant constitutes the major reaction path, as is discussed below.

\section{DISCUSSION}

4.1. Dynamical Pathways of Oxazole and Isoxazole. Combining the results of the experimental observations and the simulations, the major reaction channel for isoxazole is the ring-opening reaction arising from ON-bond cleavage. Combining the time zero shift and the fastest time constant 
of the fit to the photoelectron spectra, the timescale of the ring-opening reaction is estimated to be 35 fs. This is in good agreement with the results of the dynamics simulations, where about $80 \%$ of the trajectories undergo ring-opening within 100 fs. The reason for the fast decay is a combined barrier-free reaction path on both the $\pi \pi^{*}$-state and $\pi \sigma^{*}$-states. This is in agreement with the work of Cao, ${ }^{15}$ where most trajectories also exhibit ON-bond cleavage within the first 100 fs. Dias et al. found a major direct dissociation path in their product branching studies, which is fully consistent with our results for the bond cleavage. ${ }^{13}$ The presence of nondirect dissociation is in agreement with the long-lived process that was observed by time-resolved photoelectron spectroscopy and the $20 \%$ of the trajectories without ring-opening determined from our dynamics simulations.

The experimentally obtained timescale for the fast reaction in oxazole is about twice as large ( $85 \mathrm{fs}$ ) as that for isoxazole. By comparison with isoxazole and furan (see Section 4.2), this process can also be assigned to ring-opening dynamics. That this ring-opening reaction has a longer timescale is confirmed by the dynamics simulations, and is explained by the presence of a small barrier along the reaction path between the photoexcited $\pi \pi^{*}$ - and the reactive $\pi \sigma^{*}$-states. In oxazole, we also observe longer time constants of 2 and 65 ps. For these time constants, their DAS are unstructured (see Figure 8a), which indicates a dephased molecular wavepacket, that is, partial vibrational equilibration in the excited state. This process indicates a ring-puckering mechanism. Although the dynamical simulations cannot be extended to a ps timescale, within $100 \mathrm{fs}$ about one-third of the molecules have undergone bond cleavage. The remainder may undergo ring-puckering in the excited state at a later time.

4.2. Comparison with Similar Systems. Isoxazole and oxazole can be considered as typical 5-membered heterocyclic systems. With these data at hand, it is possible to compare them with the well-studied molecules, pyrrole and furan. The molecule that has the most similarities with oxazole and isoxazole is furan, where the $\mathrm{N}$-atom is replaced by a $\mathrm{C}-\mathrm{H}$ group. Removal of the nitrogen atom leads to a destabilization of the molecular ground state, which causes a lower-lying excited valence state and a lower ionization potential. The removal also lowers the position of the Rydberg states, which plays no role in the oxazole and isoxazole dynamics but can influence the furan dynamics (see e.g. refs 7 and 9).

For furan, recent papers indicate that the $\mathrm{O}-\mathrm{C}$ ring-opening channel is favored over ring-puckering dynamics upon excitation to its lowest excited valence state ${ }^{8,9}$ (there exist different studies favoring ring-puckering, ${ }^{39}$ but the similarities to oxazole in comparison to our calculations show the preference for ring-opening). The dynamics takes place within 60-80 fs partly because the ring-opening dynamics is superimposed with ring-puckering type of dynamics, which seem to prevent direct access to the conical intersection, similar to observations in oxazole (this timescale of ringpuckering dynamics on the ring-opening of molecules was discussed in detail in ref 40). For comparison, the time zero shifts of the photoelectron experiments of furan and oxazole are provided in Figure 7a. Here, the time zero shift of oxazole is shown in black while the furan dynamics are indicated in red. Besides an energetic shift of the signal caused by the different ionization potentials, the dynamics look rather similar, indicating similar reaction pathways.
Finally, the role of the nitrogen atom can be summarized: starting from furan, introduction of a nitrogen atom in the ring in the 2-position accelerates ring-opening and leads to a higher quantum yield of the direct opening channel, while introduction of a nitrogen atom in the 3-position disfavors ring-opening and leads to a reduced quantum yield. In a very simplistic picture, one can imagine that the electronegative nitrogen atom and oxygen atom are repelling each other while substitution in the 3-position leads to a more positively charged carbon atom, which leads to a more stable $\mathrm{C}-\mathrm{O}$ bond. This picture also explains why pyrrole and similar molecules do not predominantly undergo ring-opening reactions, as no antibonding $\sigma^{*}$-orbitals are present in the ring (see, e.g. ref 3 ). We note that the recent review by Ashfold and coworkers addresses the potential importance of $(n / \pi) \sigma^{*}$ excited states in promoting ring-opening in heterocyclic systems. ${ }^{41}$ The simple interpretation presented here is supported if the Mulliken charges for isoxazole and oxazole are analyzed. Here, in the case of isoxazole, the neighboring $\mathrm{N}$ - and $\mathrm{O}$-atoms carry small negative charges, namely -0.09 and -0.18 , respectively. The $\mathrm{C}$-atom next to the $\mathrm{O}$-atom has a positive charge of 0.05 . In the case of oxazole, the negative charges are -0.32 and -0.33 for the $\mathrm{O}$ - and $\mathrm{N}$-atoms, but the $\mathrm{C}$-atom in between has a positive charge of 0.31 . Thus, the nitrogen atom increases the positive charge of the $\mathrm{C}$-atom, which leads to a stronger $\mathrm{OC}[\mathrm{N}]$-bond and a shorter bond length, as discussed in Section 3.1.

\section{CONCLUSIONS}

Understanding the dynamical pathways of five-membered heteroaromatic systems upon light excitation remains a challenge. Several pathways are open, and the fate of the dynamics is often decided in the first few tens of femtoseconds. The theoretical challenge lies in describing the FranckCondon region and the region of the conical intersections in a balanced manner, while the experimental challenge lies in finding the right observables to decipher the ongoing dynamics. In the present contribution, we investigated two fundamental ring systems; oxazole and isoxazole.

For both systems, the dominant ultrafast reaction mechanism, besides just relaxation to the $S_{1}$ state, is ring-opening. For isoxazole, a barrier-free reaction pathway has been found, which can explain the ultrafast (sub $50 \mathrm{fs}$ ) ring-opening reaction. In oxazole, the dynamics is both about two times slower and much less efficient because of a small barrier between the bright $\pi \pi^{*}$ - and the reactive $\pi \sigma^{*}$-state, where a recent review highlighted the importance of $(n / \pi) \sigma^{*}$ excited states for promoting ring-opening in heterocyclic systems. ${ }^{41}$

Future experimental studies might include X-ray techniques such as ultrafast X-ray transient absorption spectroscopy, which recently benchmarked its applicability for studying ringopening dynamics. ${ }^{42}$

\section{ASSOCIATED CONTENT}

\section{Supporting Information}

The Supporting Information is available free of charge at https://pubs.acs.org/doi/10.1021/acs.jpca.9b11788.

The Supporting Information provides further details on the computational results, specifically vertical excitation energies; the population dynamics of the excited states; the probability of the ring-opening channel, and additional reaction mechanisms in isoxazole and oxazole (PDF) 


\section{AUTHOR INFORMATION}

\section{Corresponding Authors}

Oliver Schalk - Department of Physics, AlbaNova University Centre, Stockholm University, 10691 Stockholm, Sweden; Department of Chemistry, University of Copenhagen, DK-2100 Copenhagen, Denmark; Email: schalk.phys@gmail.com

Richard D. Thomas - Department of Physics, AlbaNova University Centre, Stockholm University, 10691 Stockholm, Sweden; 이이.org/0000-0002-9145-6366; Email: rdt@ fysik.su.se

\section{Authors}

Ting Geng - Department of Physics, AlbaNova University Centre, Stockholm University, 10691 Stockholm, Sweden

Johannes Ehrmaier - Department of Chemistry, Technical University of Munich, 85748 Garching, Germany

Gareth W. Richings - Department of Chemistry and Centre for Scientific Computing, University of Warwick, CV4 7AL Coventry, U.K.

Tony Hansson - Department of Physics, AlbaNova University Centre, Stockholm University, 10691 Stockholm, Sweden; (1) orcid.org/0000-0002-8051-0582

Graham Worth - Department of Chemistry, University College London, WC1H OAJ London, U.K.

Complete contact information is available at:

https://pubs.acs.org/10.1021/acs.jpca.9b11788

\section{Notes}

The authors declare no competing financial interest.

\section{ACKNOWLEDGMENTS}

We acknowledge the generous support and technical advice of Prof. Dr. Raimund Feifel. This work was supported by the Swedish Research Council (VR), the Knut and Alice Wallenberg Foundation, and the Wenner-Gren Foundation. G.W.R. acknowledges financial support from the Leverhulme Trust (RPG-2016-055) and EPSRC (EP/S028986/1).

\section{REFERENCES}

(1) Roberts, G. M.; Williams, C. A.; Yu, H.; Chatterley, A. S.; Young, J. D.; Ullrich, S.; Stavros, V. G. Probing Ultrafast Dynamics in Photoexcited Pyrrole: Timescales for $1 \pi \sigma^{*}$ Mediated $\mathrm{H}$-atom Elimination. Faraday Discuss. 2013, 163, 95-116.

(2) Neville, S. P.; Kirkby, O. M.; Kaltsoyannis, N.; Worth, G. A.; Fielding, H. H. Identification of a New Electron-transfer Relaxation Pathway in Photoexcited Pyrrole Dimers. Nat. Commun. 2016, 7, 11357.

(3) Wu, G.; Neville, S. P.; Schalk, O.; Sekikawa, T.; Ashfold, M. N. R.; Worth, G. A.; Stolow, A. Excited State Non-adiabatic Dynamics of Pyrrole: A Time-resolved Photoelectron Spectroscopy and Quantum Dynamics Study. J. Chem. Phys. 2015, 142, 074302.

(4) Geng, T.; Schalk, O.; Neville, S. P.; Hansson, T.; Thomas, R. D. Dynamics in Higher Lying Excited States: Valence to Rydberg Transitions in the Relaxation Paths of Pyrrole and Methylated Derivatives. J. Chem. Phys. 2017, 146, 144307.

(5) Montero, R.; León, I.; Fernández, J. A.; Longarte, A. Femtosecond Excited State Dynamics of Size Selected Neutral Molecular Clusters. J. Phys. Chem. Lett. 2016, 7, 2797-2802.

(6) Kirkby, O. M.; Parkes, M. A.; Neville, S. P.; Worth, G. A.; Fielding, H. H. Non-radiative Relaxation Dynamics of Pyrrole Following Excitation in the Range 249.5-200 nm. Chem. Phys. Lett. 2017, 683, 179-185.

(7) Spesyvtsev, R.; Horio, T.; Suzuki, Y.-I.; Suzuki, T. Excited-state Dynamics of Furan Studied by Sub-20-fs Time-resolved Photo- electron Imaging Using 159-nm Pulses. J. Chem. Phys. 2015, 143, 014302 .

(8) Oesterling, S.; Schalk, O.; Geng, T.; Thomas, R. D.; Hansson, T.; de Vivie-Riedle, R. Substituent Effects on the Relaxation Dynamics of Furan, Furfural and $\beta$-Furfural: A Combined Theoretical and Experimental Approach. Phys. Chem. Chem. Phys. 2017, 19, 20252035.

(9) Schalk, O.; Geng, T.; Hansson, T.; Thomas, R. D. The Ringopening Channel and the Influence of Rydberg States on the Excited State Dynamics of Furan and its Derivatives. J. Chem. Phys. 2018, 149, 084303.

(10) Schalk, O.; Boguslavskiy, A. E.; Stolow, A. Substituent Effects on Dynamics at Conical Intersections: Cyclopentadienes. J. Phys. Chem. A 2010, 114, 4058-4064.

(11) Nunes, C. M.; Reva, I.; Pinho e Melo, T. M. V. D.; Fausto, R. UV-Laser Photochemistry of Isoxazole Isolated in a Low-Temperature Matrix. J. Org. Chem. 2012, 77, 8723-8732.

(12) Nunes, C. M.; Reva, I.; Fausto, R. Capture of an Elusive Nitrile Ylide As an Intermediate in Isoxazole-Oxazole Photoisomerization. $J$. Org. Chem. 2013, 78, 10657-10665.

(13) Dias, N.; Joalland, B.; Ariyasingha, N. M.; Suits, A. G.; Broderick, B. M. Direct Versus Indirect Photodissociation of Isoxazole From Product Branching: A Chirped-pulse Fourier Transform mmWave Spectroscopy/Pulsed Uniform Flow Investigation. J. Phys. Chem. A 2018, 122, 7523-7531.

(14) Su, M.-D. Mechanistic Analysis of an Isoxazole-oxazole Photoisomerization Reaction Using a Conical Intersection. J. Phys. Chem. A 2015, 119, 9666-9669.

(15) Cao, J. Photoinduced Reactions of Both 2-Formyl-2H-azirine and Isoxazole: A Theoretical Study Based on Electronic Structure Calculations and Nonadiabatic Dynamics Simulations. J. Chem. Phys. 2015, 142, 244302.

(16) Tanaka, H.; Matsushita, T.; Nishimoto, K. Theoretical Study on the Photochemical Transposition Reaction of Oxazole. J. Am. Chem. Soc. 1983, 105, 1753-1760.

(17) Cao, J.; Xie, Z.-Z.; Yu, X. Excited-state Dynamics of Oxazole: A Combined Electronic Structure Calculations and Dynamic Simulations Study. Chem. Phys. 2016, 474, 25-35.

(18) Maeda, M.; Kojima, M. Photorearrangements of Phenyloxazoles. J. Chem. Soc., Perkin Trans. 1 1977, 239-247.

(19) Walker, I. C.; Palmer, M. H.; Delwiche, J.; Hoffmann, S. V.; Vieora, P. L.; Mason, N. J.; Guest, M. F.; Hubin-Franskin, M.-J.; Heinesch, J.; Giuliani, A. The Electronic States of Isoxazole Studied by VUV Absorption, Electron Energy-loss Spectroscopies and $\mathrm{Ab}$ Initio Multi-reference Configuration Interaction Calculations. Chem. Phys. 2004, 297, 289-306.

(20) Palmer, M. H.; Ganzenmüller, G.; Walker, I. C. The Electronic States of Oxazole Studied by VUV Absorption and Electron Energyloss (Eel) Spectroscopies, and Ab Initio Configuration Interaction Methods. Chem. Phys. 2007, 334, 154-166.

(21) Ullman, E. F.; Singh, B. Photochemical Transposition of Ring Atoms in Five-membered Heterocycles. The Photorearrangement of 3,5-Diphenylisoxazole. J. Am. Chem. Soc. 1966, 88, 1844-1845.

(22) Kłoda, T.; Matsuda, A.; Karlsson, H. O.; Elshakre, M.; Linusson, P.; Eland, J. H. D.; Feifel, R.; Hansson, T. Strong-field Photoionization of $\mathrm{O}_{2}$ at Intermediate Light Intensity. Phys. Rev. A: At., Mol., Opt. Phys. 2010, 82, 033431.

(23) Schalk, O.; Stenrup, M.; Geng, T.; Lindh, R.; Thomas, R. D.; Feifel, R.; Hansson, T. Influence of Alkoxy Groups on the Photoinduced Dynamics of Organic Molecules Exemplified on Alkyl Vinyl Ethers. J. Phys. Chem. A 2015, 119, 11105-11112.

(24) Schalk, O.; Boguslavskiy, A. E.; Stolow, A.; Schuurman, M. S. Through-bond Interactions and the Localization of Excited-state Dynamics. J. Am. Chem. Soc. 2011, 133, 16451-16458.

(25) Schalk, O.; Schuurman, M. S.; Wu, G.; Lang, P.; Mucke, M.; Feifel, R.; Stolow, A. Internal Conversion Versus Intersystem Crossing: What Drives the Gas Phase Dynamics of Cyclic $\alpha, \beta$ Enones? J. Phys. Chem. A 2014, 118, 2279-2287. 
(26) Wolf, T. J. A.; Kuhlman, T. S.; Schalk, O.; Martínez, T. J.; Møller, K. B.; Stolow, A.; Unterreiner, A.-N. Hexamethylcyclopentadiene: Time-resolved Photoelectron Spectroscopy and $\mathrm{Ab}$ Initio Multiple Spawning Simulations. Phys. Chem. Chem. Phys. 2014, 16, 11770-11779.

(27) Schalk, O.; Geng, T.; Thompson, T.; Baluyot, N.; Thomas, R. D.; Tapavicza, E.; Hansson, T. Cyclohexadiene Revisited: A Timeresolved Photoelectron Spectroscopy and Ab Initio Study. J. Phys. Chem. A 2016, 120, 2320-2329.

(28) Møller, C.; Plesset, M. S. Note on an Approximation Treatment for Many-electron Systems. Phys. Rev. 1934, 46, 618-622.

(29) Schirmer, J. Beyond the Random-phase Approximation: A New Approximation Scheme for the Polarization Propagator. Phys. Rev. A: At., Mol., Opt. Phys. 1982, 26, 2395-2416.

(30) Sapunar, M.; Ponzi, A.; Chaiwongwattana, S.; Mališ, M.; Prlj, A.; Decleva, P.; Došlić, N. Timescales of N-H Bond Dissociation in Pyrrole: A Nonadiabatic Dynamics Study. Phys. Chem. Chem. Phys. 2015, 17, 19012-19020.

(31) Belyaev, A. K.; Domcke, W.; Lasser, C.; Trigila, G. Nonadiabatic Nuclear Dynamics of the Ammonia Cation Studied by Surface Hopping Classical Trajectory Calculations. J. Chem. Phys. 2015, 142, 104307.

(32) Xie, W.; Domcke, W. Accuracy of Trajectory Surface-hopping Methods: Test for a Two-dimensional Model of the Photodissociation of Phenol. J. Chem. Phys. 2017, 147, 184114.

(33) TURBOMOLE V7.2 2017, a Development of University of Karlsruhe and Forschungszentrum Karlsruhe GmbH; 1989-2007, TURBOMOLE GmbH, since 2007; available from http://www. turbomole.com.

(34) Dunning, T. H., Jr. Gaussian Basis Sets for Use in Correlated Molecular Calculations. I. The Atoms Boron Through Neon and Hydrogen. J. Chem. Phys. 1989, 90, 1007-1023.

(35) Stiefvater, O. L. The Complete Structure of Isoxazole From Naturally Occurring Isotopic Forms by Double Resonance Modulated Microwave Spectroscopy. J. Chem. Phys. 1975, 63, 2560-2569.

(36) Kumar, A.; Sheridan, J.; Stiefvater, O. L. The Microwave Spectrum of Oxazole I. The Complete Structure by Drm Microwave Spectroscopy. Z. Naturforsch., A: Phys., Phys. Chem., Kosmophys. 1978, 33, 145-152.

(37) Palmer, M. H.; Findlay, R. H.; Egdell, R. G. The Electronic Structure of Heteroaromatic Molecules; Ab Initio Calculations and Photoelectron Spectra for the Isomeric-Oxazoles and Some -Oxadiazoles. J. Mol. Struct. 1977, 40, 191-210.

(38) Stolow, A.; Underwood, J. G. Time-resolved Photoelectron Spectroscopy of Non-adiabatic Dynamics in Polyatomic Molecules. Adv. Phys. Chem. 2008, 139, 497-584.

(39) Adachi, S.; Schatteburg, T.; Humeniuk, A.; Mitrić, R.; Suzuki, T. Probing Ultrafast Dynamics During and after Passing Through Conical Intersections. Phys. Chem. Chem. Phys. 2019, 21, 1390213905.

(40) Schalk, O.; Galiana, J.; Geng, T.; Larsson, T. L.; Thomas, R. D.; Fdez. Galván, I.; Hansson, T.; Vacher, M. Competition Between Ringpuckering and Ring-opening Excited State Reactions Exemplified on 5H-Furan-2-one and Derivatives. J. Chem. Phys. 2020, 152, 064301.

(41) Ashfold, M. N. R.; Bain, M.; Hansen, C. S.; Ingle, R. A.; Karsili, T. N. V.; Marchetti, B.; Murdock, D. Exploring the Dynamics of the Photoinduced Ring-Opening of Heterocyclic Molecules. J. Phys. Chem. Lett. 2017, 8, 3440-3451.

(42) Bhattacherjee, A.; Schnorr, K.; Oesterling, S.; Yang, Z.; Xue, T.; de Vivie-Riedle, R.; Leone, S. R. Photoinduced Heterocyclic Ring Opening of Furfural: Distinct Open-chain Product Identification by Ultrafast X-Ray Transient Absorption Spectroscopy. J. Am. Chem. Soc. 2018, 140, 12538-12544. 